\title{
PA-080 CRYPTOCOCCAL MENINGOENCEPHALITIS IN HIV-INFECTED PATIENTS IN MADAGASCAR: HIGH PREVALENCE AND LETHALITY AND THERAPEUTIC CHALLENGES
}

Rivo Rakotoarivelo, ${ }^{1}$ Mihaja Raberahona, ${ }^{2}$ Njary Randriamampionona, ${ }^{3}$ Rabezanahary Andriamihaja, ${ }_{1}^{4}$ Tahinamandranto Rasamoelina, ${ }^{3}$ Muriel Cornet, ${ }^{5}$ Mamy Jean De Dieu Randria, ${ }^{2}$ Thomas Benet, ${ }^{6}$ Philippe Vanhems, ${ }^{6}$ Mala Rakoto Andrianarivelo ${ }^{3} .{ }^{1} \mathrm{CHU}$ Tambohobe Fianarantsoa, Madagascar; ${ }^{2} \mathrm{HUJR}$ de Befelatanana, Madagascar; ${ }^{3} \mathrm{CICM}$, Madagascar; ${ }^{4} \mathrm{CHU}$ Analakininina, Madagascar; ${ }^{5} \mathrm{CHU}$ de Grenoble, France; ${ }^{6} \mathrm{HEH}$, France

\subsection{6/bmjgh-2016-000260.112}

Background In Madagascar the epidemiology of cryptococcosis is poorly documented. The main objective of this study was to estimate the prevalence of Cryptococcal meningoencephalitis (CM) in Madagascar and to describe the presentation of the cases.

Methods This is an observational transverse study conducted in the hospitals of Antananarivo and Toamasina cities. Between 3 November 2014 and 8 June 2016, HIV-infected adults presenting CD4 cell count $\leq 200 / \mathrm{mm} 3$ were selected. The crytococcocal antigen (CrAg) was screened in the blood using a lateral flow immunoassay (IMMYCrAg ${ }^{\circledR}$ LFA). If the result was positive and the patient symptomatic, CrAg was checked in the cerebrospinal fluid (CSF) and examined with India ink, and culture was performed. The isolated strains were subsequently analysed using MALDI-TOF and an antifungal susceptibility test was performed using the E-test method (BioMérieux).

Results Overall, 118 patients were included. The mean CD4 cell count was $86.4 / \mathrm{mm} 3(\mathrm{SD} \pm 60.6)$ and $35.6 \%$ of the patients were under ARV therapy at baseline. HIV-1 viral load of $88.5 \%$ of patients was positive. We compared the clinical characteristics of patients with cryptococcal infections to those of controls without $\mathrm{CM}$. Eleven cases of CM were identified corresponding to a prevalence of 15.1\% (95\% CI: 7.8-25.4\%). Cryptococcus neoformans var. grubii (serotype A) was isolated. Fever, headache, neck pain 
and night sweats were the most common signs. In 7 cases, $\mathrm{CrAg}$ titres in the CSF were very high $(\geq 2560)$ and did not decrease even 2 months post-treatment. The Case Fatality Rate was unacceptably high (69\%).

Conclusions Overall, prevalence of cryptococcal meningoencephalitis (CM) in Madagascar was very high (15.1\%) compared to that observed in some Sub-Saharan African countries. The point-of-care LFA CrAg test was confirmed to be reliable and cost-effective for the diagnosis. Challenges to facilitate access to more effective molecules to treat patients with CM include heavy administrative formalities linked to drug importation and low level of priority in implementing the national control programme. 